\title{
Prognostic significance of sirtuin 2 protein nuclear localization in glioma: An immunohistochemical study
}

\author{
NATSUKO IMAOKA ${ }^{1,2}$, MASAHARU HIRATSUKA ${ }^{4}$, MITSUHIKO OSAKI $^{1,5}$, HIDEKI KAMITANI $^{3}$, \\ ATSUSHI KAMBE ${ }^{3}$, JUNYA FUKUOKA ${ }^{6}$, MASANORI KURIMOTO $^{7}$, SHOICHI NAGAI $^{7}$, \\ FUTOSHI OKADA ${ }^{5}$, TAKASHI WATANABE ${ }^{3}$, EISAKU OHAMA ${ }^{2}$, \\ SHINSUKE KATO ${ }^{2}$ and MITSUO OSHIMURA ${ }^{1,4}$
}

\begin{abstract}
${ }^{1}$ Department of Biomedical Science, Institute of Regenerative Medicine and Biofunction, Graduate School of Medical Science, Tottori University, Divisions of ${ }^{2}$ Neuropathology and ${ }^{3}$ Neurosurgery, Department of Brain and Neurosciences, Divisions of ${ }^{4}$ Molecular and Cell Genetics and ${ }^{5}$ Pathological Biochemistry, School of Life Science, Tottori University Faculty of Medicine, Yonago, Tottori 683-8503; ${ }^{6}$ Department of Surgical Pathology, Toyama University Hospital, and

${ }^{7}$ Department of Neurosurgery, Faculty of Medicine, University of Toyama, Sugitani, Toyama 930-0194, Japan
\end{abstract}

Received March 8, 2012; Accepted April 17, 2012

DOI: $10.3892 /$ or.2012.1872

\begin{abstract}
The sirtuin 2 (SIRT2) protein is a member of the sirtuin family and homologous to Sir2 (silent information regulator 2) of Saccharomyces cerevisiae. To assess the pathobiological significance of SIRT2 protein expression and/or subcellular localization in human glioma, we examined SIRT2 protein expression in human gliomas using a polyclonal anti-SIRT2 antibody and immunohistochemistry. In this study, samples from 23 patients with glioblastoma (GB, grade IV), 8 patients with diffuse astrocytoma (DA, grade II) and 5 healthy individuals were examined. We established a SIRT2 labeling index (SIRT2-LI) that represents the percentage of cells with SIRT2 localized to the nucleus. The mean SIRT2-LI was $65.8 \pm 18.6$ in GB samples, $41.2 \pm 22.8$ in DA samples, and $28.6 \pm 12.3$ in normal control samples. The SIRT2-LI of GB samples was significantly higher than that of normal control samples $(\mathrm{P}<0.01$, MannWhitney's U-test) and that of DA samples $(\mathrm{P}<0.05)$. Moreover, the SIRT2-LI was positively correlated with malignant progression. Specifically, samples from patients with GB were divided into two groups, low SIRT2-LI $(<60 \%)$ and high SIRT2-LI $(\geq 60 \%$ ), and the patients with low SIRT2-LI samples survived significantly longer than patients with high SIRT2-LI samples $(\mathrm{P}<0.05$, Kaplan-Meier method and log-rank test). In conclusion, SIRT2-LI was indicative of glioma malignancy, and it may be predictive of GB patient survival.
\end{abstract}

Correspondence to: Dr Mitsuo Oshimura, Department of Biomedical Science, Institute of Regenerative Medicine and Biofunction, Graduate School of Medical Science, Tottori University, 86 Nishi-cho, Yonago, Tottori 683-8503, Japan

E-mail: oshimura@med.tottori-u.ac.jp

Key words: sirtuin 2 protein, glioblastoma, immunohistochemistry, survival, malignancy

\section{Introduction}

Gliomagenesis, like development of other malignancies, involves the accumulation of a series of genetic alterations (1). Many of the genes altered during glioma development were identified using standard molecular approaches, and these genes normally participate in a range of cellular functions (e.g., governing cellular proliferation, cell infiltration, angiogenesis, and cell death). Genetic aberrations are frequently found in human glioma: gene amplification of epidermal growth factor receptor (EGFR) (2) and murine double minute 2 (MDM2) $(3,4)$; overexpression of platelet-derived growth factor receptor (PDGFR) (5); gene mutation of retinoblastoma (Rb), p53 (6) and phosphatase and tensin homolog deleted on chromosome ten (PTEN) (7); deletion of cyclin-dependent kinase inhibitor 2A (CDKN2A/p16INK4A) (3,4). Despite this information, mechanism of tumorigenesis and progression in glioblastoma (GB) have not been understood in detail because malignant gliomas, including GB, have significant morphological heterogeneity in each tumor; individual tumors are genetically and histopathologically very heterogeneous. In order to overcome this complexity in glioma phenotypes and identify putative therapeutic targets, more global and systematic approaches, including proteomic (8), transcriptomic (5,6), and comparative genomic hybridization analyses, have been performed. Under these circumstances, we performed proteomic analysis to compare protein expression profiles in diffuse astrocytoma (DA) and GB, and we found that total expression of SIRT2 was lower in GB than in DA (8).

Sirtuin 2 (SIRT2) is a NAD-dependent deacetylase, and is a member of the human sirtuin family that was initially identified based on structural homology to the Saccharomyces cerevisiae Sir2 protein (silent information regulator) (9). In human, there are seven proteins of the sirtuin family (SIRT1-7) $(10,11)$. Among all sirtuins, SIRT2 was the most highly express in brain tissue, and SIRT2 expression was particularly prominent in the postnatal hippocampus (12), and it has been suggested that SIRT2 


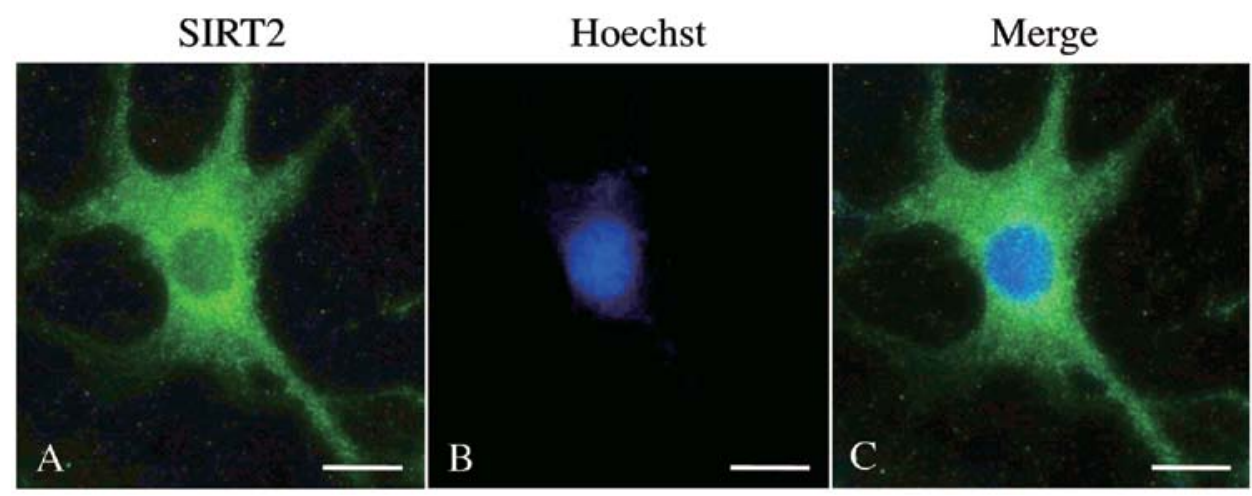

Figure 1. Anti-SIRT2 immunofluorescence of primary glial cell isolated from C57BL/6 mouse. (A) Anti-SIRT2 staining in a glial cell; cytoplasmic staining is observed. (B) Hoechst counterstaining. (C) Merged image. Bar, $10 \mu \mathrm{m}$.

has neuronal functions, including cytoskeletal growth cone dynamics (11), neurite outgrowth, and oligodendrocyte arborization in vitro (13). It has been suggested that SIRT2 may have tumor-suppressor activity because SIRT2 suppressed colony formation in glioma cell lines and controlled cell cycle progression by acting as a regulator of mitotic exit (14-16). Additionally, we reported that subcellular localization of SIRT2 was translocated from cytoplasm to nucleus when cells were exposed to ionizing radiation in the human fibroblast cell line TIG-1 (16). In the present study, we evaluated of the expression and subcellular localization of SIRT2 in samples from patients with GB and/ or DA using immunohistochemistry, and we assessed the prognostic significance of SIRT2 expression pattern in GB patients. We demonstrated that although nuclear SIRT2 expression was seen in all gliomas examined, SIRT2 localization was predominantly nuclear in GB samples but predominantly cytoplasmic in control samples; moreover, the percentage of GB cells with SIRT2-positive nuclei was negatively correlated with survival time of patients with GB.

\section{Materials and methods}

Cell culture. As a control, we analyzed primary glial cells isolated from brain tissue of C57BL/6 mice. Cells were grown in Dulbecco's modified Eagle's medium (DMEM, Gibco Invitrogen Corp., Carlsbad, CA, USA) supplemented with $10 \%$ fetal bovine serum, at $37^{\circ} \mathrm{C}$, under $5 \% \mathrm{CO}_{2}$, and in 12 -well chamber slides (17).

Tissue collections. This study used surgically resected samples from 16 patients with glioma being treated at Tottori University and from 15 patients with glioma whose samples were stored at the tissue archive of Toyama University Hospital (Table I). The samples were fixed with $10 \%$ formalin and embedded in paraffin. The glioma specimens were classified according to the World Health Organization (WHO) International Histological Classification of Tumors (18). We also examined autopsy specimens of brain tissue from 5 neurologically and neuropathologically normal individuals (causes of death: acute heart failure, squamous cell carcinoma, acute myocardial infarction, disseminated intravascular coagulation, or pneumonia). Among 31 brain tumor samples, 15 samples of GB (patient nos. 10-24 in Table I) were subjected to the tissue microarray (TMA) method, in which tissue cylinders with a diameter of $0.6 \mathrm{~mm}$ were punched from GB areas of each tissue blocks (19) (Table I). Clinical data, including age, gender, and survival time from the initial operation, were obtained from the hospital records. Multiple 5- $\mu \mathrm{m}$ sections were prepared from each specimen. One section was stained with hematoxylin and eosin (H\&E), and the others were used for the immunohistochemical tests. This study was approved by the Ethics Committer of Tottori University (Permission: no. 1434) and Toyama University Hospital (Permission: no. 19-12).

Immunofluorescence. Primary glial cells grown in 12-well chamber slides were washed twice in phosphate-buffered saline, $\mathrm{pH} 7.4$ (PBS); fixed in 4\% paraformaldehyde for $15 \mathrm{~min}$; and permeabilized in $0.2 \%$ Nonidet P-40 (Nacalai Tesque, Kyoto, Japan) in PBS for $2 \mathrm{~min}$. After two sequential 5-min washes in PBS, cells were incubated in PBS with 5\% skim milk (Difco, Detroit, MI, USA) for $30 \mathrm{~min}$. Normal serum served as blocking reagent. A rabbit polyclonal antibody raised against purified, recombinant human SIRT2 protein was used at a 1:100 dilution; the antibody was diluted in PBS containing 1\% bovine serum albumin. The specificity and affinity of the polyclonal anti-human-SIRT2 antibody (anti-SIRT2) (Santa Cruz Biotechnology Inc., Santa Cruz, CA, USA) for use in primary cell cultures was established previously by using the anti-SIRT2 antibody to detect SIRT2 in mouse cells (20). Herein, cells were incubated with anti-SIRT2 antibody for $1 \mathrm{~h}$ at room temperature. Thereafter, they were washed three times for $5 \mathrm{~min}$ each in PBS with $0.2 \%$ Nonidet P-40, then incubated in PBS with 5\% skim milk for $15 \mathrm{~min}$, and finally incubated with Alexa Flour 488 goat anti-rabbit IgG (Invitrogen Corp., Carlsbad, CA, USA) diluted 1:1,000 for $30 \mathrm{~min}$. Stained cells were washed three times for 5 min each in PBS with $0.2 \%$ Nonidet P-40 and then counterstained with $1 \mu \mathrm{g} / \mathrm{ml}$ Hoechst 33258 (Sigma-Aldrich Inc., St. Louis, MO, USA). PBS replaced the anti-SIRT2 antibody in parallel negative-control experiments.

Immunohistochemistry. Sections were deparaffinized, and endogenous peroxidase activity was quenched by incubation for 30 min with $0.3 \%$ hydrogen peroxide, and samples were washed with PBS. Normal serum served as blocking reagent. The anti-SIRT2 antibody was diluted in PBS with $1 \%$ bovine serum and used at a dilution of 1:250. The specificity and 
Table I. Characteristics of 23 patients with glioblastoma, eight patients with astrocytoma, and five normal individuals.

\begin{tabular}{|c|c|c|c|c|c|c|c|}
\hline Patient no. & Age & Gender & $\begin{array}{c}\text { Diagnosis } \\
\text { (WHO grade) }\end{array}$ & $\begin{array}{l}\text { Tissue } \\
\text { sample }\end{array}$ & $\begin{array}{c}\text { SIRT2 } \\
\text { labeling } \\
\text { index }(\%)\end{array}$ & $\begin{array}{c}\text { SIRT2 } \\
\text { cytoplasm }\end{array}$ & $\begin{array}{l}\text { Survival } \\
\text { (months) }\end{array}$ \\
\hline
\end{tabular}

\begin{tabular}{|c|c|c|c|c|c|c|c|}
\hline \multicolumn{8}{|c|}{ Brain tumor } \\
\hline 1 & 70 & Female & GB (IV) & Biopsy & 72.3 & - & 9 \\
\hline 2 & 26 & Female & GB (lV) & Biopsy & 75.3 & - & 30 \\
\hline 3 & 56 & Male & GB (IV) & Biopsy & 69.4 & + & 17 \\
\hline 4 & 57 & Male & GB (lV) & Biopsy & 60.6 & + & 17 \\
\hline 5 & 58 & Female & GB (lV) & Biopsy & 68.8 & + & 34 \\
\hline 6 & 55 & Male & GB (IV) & Biopsy & 75.2 & + & 4 \\
\hline 7 & 65 & Male & GB (lV) & Biopsy & 91.8 & + & 16 \\
\hline 8 & 76 & Female & GB (lV) & Biopsy & 74.9 & - & 5 \\
\hline 9 & 71 & Male & GB (lV) & TMA & 39.9 & - & 26 \\
\hline 10 & 50 & Male & GB (IV) & TMA & 63.1 & + & 4 \\
\hline 11 & 72 & Female & GB (lV) & TMA & 89.1 & - & 5 \\
\hline 12 & 62 & Male & GB (lV) & TMA & 66.3 & + & 14 \\
\hline 13 & 78 & Female & GB (lV) & TMA & 69.5 & + & 15 \\
\hline 14 & 32 & Female & GB (lV) & TMA & 74.4 & - & 12 \\
\hline 15 & 58 & Female & GB (lV) & TMA & 22.8 & + & 8 \\
\hline 16 & 58 & Female & GB (lV) & TMA & 90.4 & - & 6 \\
\hline 17 & 58 & Male & GB (IV) & TMA & 96.6 & - & 24 \\
\hline 18 & 67 & Male & GB (IV) & TMA & 51.7 & - & 46 \\
\hline 19 & 49 & Male & GB (lV) & TMA & 56.1 & - & 17 \\
\hline 20 & 69 & Male & GB (lV) & TMA & 49.7 & + & 8 \\
\hline 21 & 69 & Male & GB (lV) & TMA & 28.8 & - & 27 \\
\hline 22 & 63 & Male & GB (lV) & TMA & 52.0 & + & 12 \\
\hline 23 & 71 & Male & GB (lV) & TMA & 75.1 & - & 6 \\
\hline 24 & 51 & Female & DA (ll) & Biopsy & 40.5 & - & 103 \\
\hline 25 & 68 & Male & DA (ll) & Biopsy & 34.8 & + & 7 \\
\hline 26 & 25 & Male & DA (ll) & Biopsy & 26.7 & + & 56 \\
\hline 27 & 20 & Male & DA (ll) & Biopsy & 19.4 & + & 36 \\
\hline 28 & 25 & Male & DA (ll) & Biopsy & 34.2 & - & 58 \\
\hline 29 & 18 & Female & DA (ll) & Biopsy & 17.4 & + & 41 \\
\hline 30 & 30 & Female & DA (ll) & Biopsy & 73.0 & + & 25 \\
\hline 31 & 74 & Male & DA (11) & Biopsy & 83.8 & + & 9 \\
\hline Normal & & & ause of dea & & & & \\
\hline 32 & 70 & Female & AHF & Autopsy & 25.5 & + & - \\
\hline 33 & 76 & Male & SCC & Autopsy & 12.8 & + & - \\
\hline 34 & 77 & Male & AMI & Autopsy & 25.2 & + & - \\
\hline 35 & 78 & Male & DIC & Autopsy & 29.0 & + & - \\
\hline 36 & 76 & Male & $\mathrm{Pn}$ & Autopsy & 50.6 & + & - \\
\hline
\end{tabular}

GB, glioblastoma; DA, diffuse astrocytoma; TMA, tissue microarray; AHF, Acute heart failure; SCC, squamous cell carcinoma (external acoustic meatus); AMI, acute myocardinal infarction; DIC, disseminated intravascular coagulation; Pn, pneumonia.

affinity of anti-SIRT2 for use in sectioned tissue samples was established previously by using the anti-SIRT2 antibody for immunohistochemical detection of SIRT2 in paraffin sections (20). Sections were incubated with the anti-SIRT2 antibody for $18 \mathrm{~h}$ at $4^{\circ} \mathrm{C}$. PBS replaced the antibody in parallel negativecontrol samples. The EnVision kit (Dako, Glostrup, Denmark) was used according to the manufacturer's protocol to detect the bound antibody. 3,3'-Diaminobenzidene tetrahydrochloride 

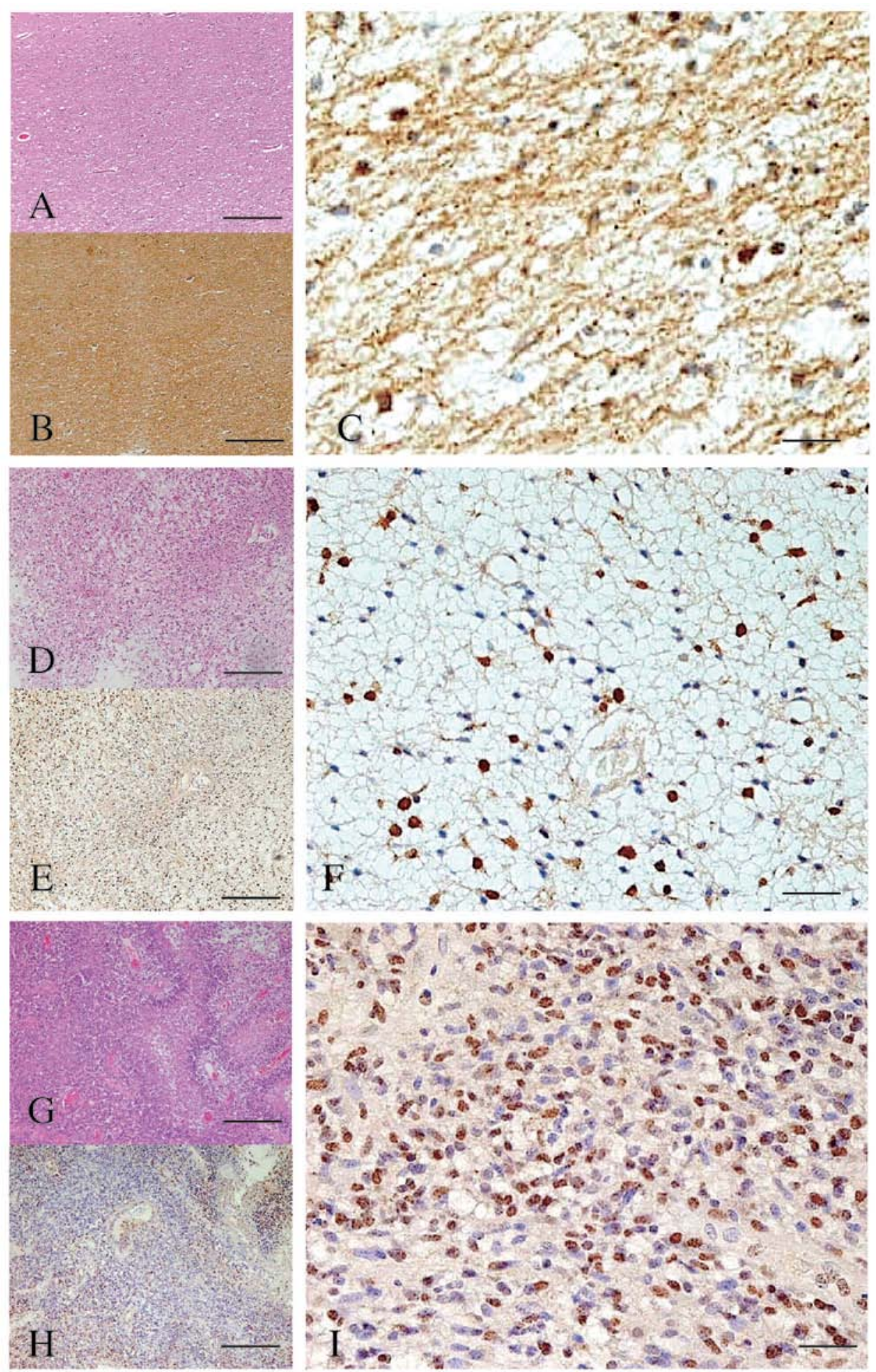

Figure 2. Immunohistochemistry of normal brain tissue and glioma using the anti-SIRT2 antibody. (A-C) Normal brain tissue. Serial sections of normal frontal white matter stained with hematoxylin and eosin (H\&E, A) and anti-SIRT2 antibody (B). At high-power magnification B, 28.6\% of the astrocytic cells have positively-stained cytoplasm (C). (D-F) Diffuse astrocytoma (grade II). Contiguous sections of a diffuse astrocytoma stained with H\&E (D) and anti-SIRT2 antibody (E). At high-power magnification of panel E, 37\% of the astrocytic tumor cells have nuclear staining (F). (G-I) Glioblastoma (GB, grade IV). Contiguous sections of GB stained with $\mathrm{H} \& \mathrm{E}$ (G) and anti-SIRT2 antibody (H). At high-power magnification $\mathrm{H}, \sim 74 \%$ glioblastoma cells have positively-stained nuclei (I). Bars in panel A, B, D, E, G and $\mathrm{H}=160 \mu \mathrm{m}$; Bar in panel C, F and I $=40 \mu \mathrm{m}$.

(DAB) was the final chromogen. Sections were counterstained with hematoxylin. More than 200 tumor cells in the tumor area or astrocytes in normal brain were scored, and the percentage of cells showing positive staining in nuclei was designated as the SIRT2 labeling index (SIRT2-LI), as a percentage (\%). SIRT2 expression in cytoplasm was also evaluated and classified into two groups; negative (-), when no immunoreactivity was observed in cytoplasm in tumor cells in glioma specimens or astrocytes in normal brain specimens, positive (+), when immunoreactivity was observed in the cytoplasm without regard to 

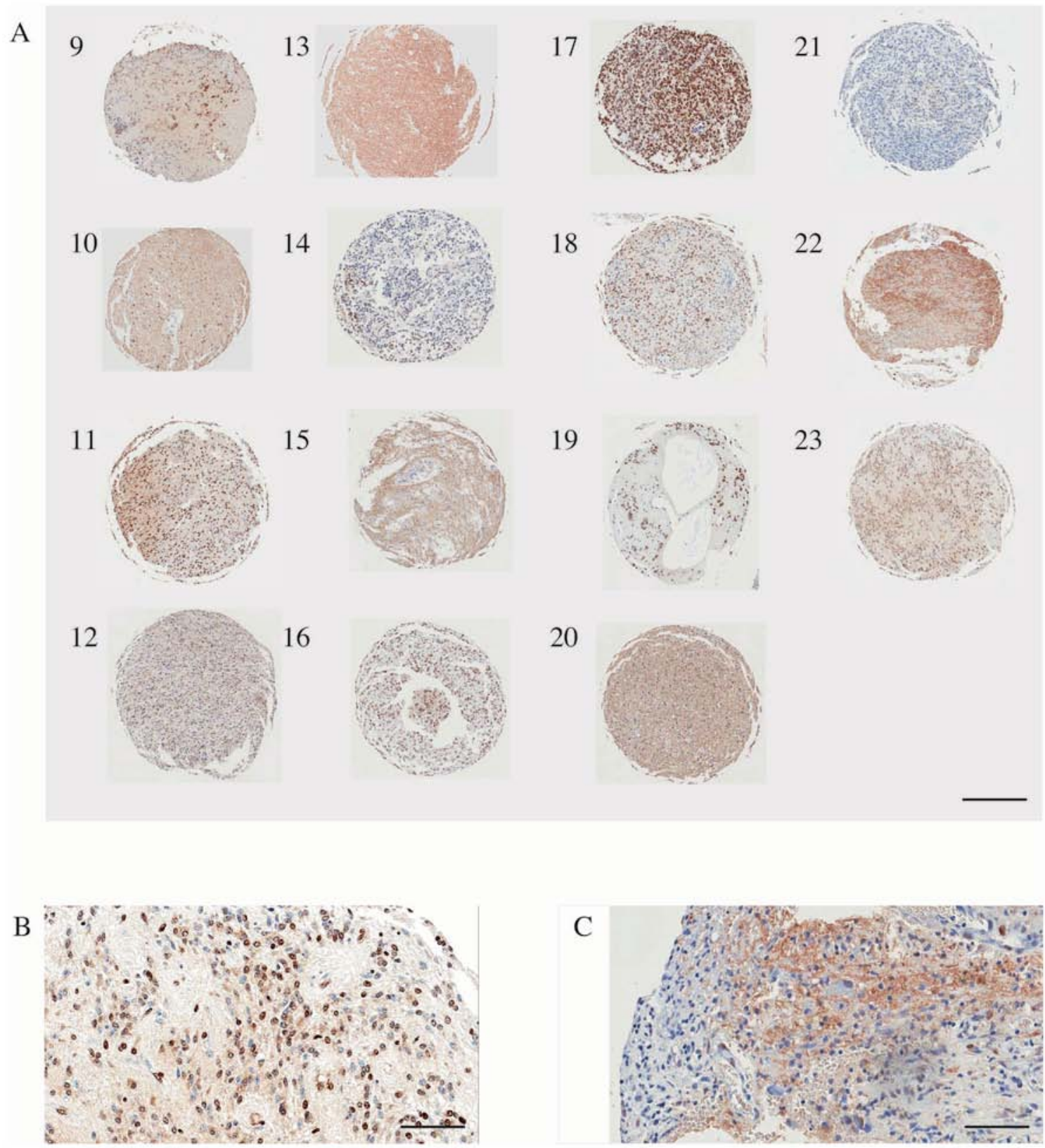

Figure 3. Tissue microarray (TMA) of GB patients. (A) Implant specimens from patients with GB were immunostained with the anti-SIRT2 antibody (patient nos. 10-24 in Table I). (B) High-power magnification images of samples from GB patient no. 12 in Table I. (C) High-power magnification images of samples from GB patient no. 18 in Table I. Bar in A, $300 \mu \mathrm{m}$; Bars in B and C, $100 \mu \mathrm{m}$.

percentage of positive cells. SIRT2 cytoplasm positive rate (\%) was calculated as follows: positive case number/total case number in GB, DA and normal control, respectively.

Statistical analysis. Mann-Whitney's U-test was used to compare nuclear SIRT2-LI in GB, DA, and normal control samples. The survival curve was estimated by the Kaplan-Meier method and log-rank test. $\mathrm{P}<0.05$ was considered significant.

\section{Results}

Immunofluorescence and immunohistochemistry of SIRT2. No antibody staining was seen in cells treated with PBS rather than anti-SIRT2 antibody (negative controls) in the immunofluorescent or immunohistochemical studies. As expected
(10), anti-SIRT2 antibody staining localized to the cytoplasm in astrocytic cells from primary cultures of normal mouse brain, but no significant reaction was seen in the nucleus of these cells (Fig. 1). Similarly, anti-SIRT2 antibody staining was observed in the cytoplasm of some astrocytes in autopsy samples from the normal individuals, although the cytoplasmic staining varied from cell to cell (Fig. 2A-C). Nuclear staining was also seen in a small percentage of astrocytes in autopsy samples (Fig. 2C). The signal intensity and proportion of positively stained glioma cells varied with histological grade. A representative stained section from a DA (grade II) is shown in Fig. 2D-F, and a specimen from a GB (grade IV) is shown in Fig. 2G-I and Fig. 3, respectively. Clear immunoreactivity was also observed in TMA specimens (Fig. 3). The mean SIRT2-LI for all specimens within each group was 
A

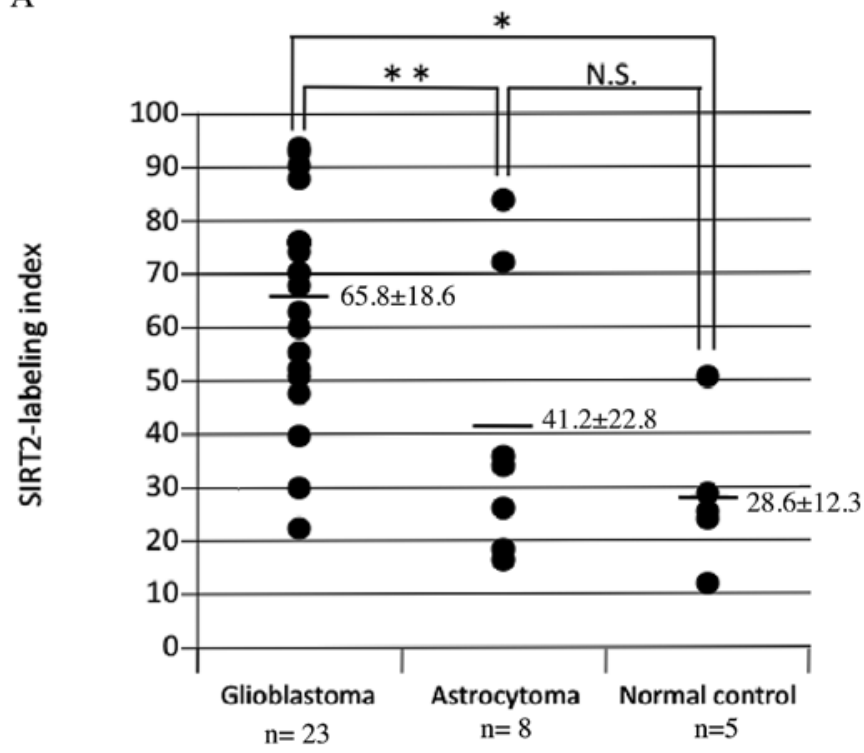

B

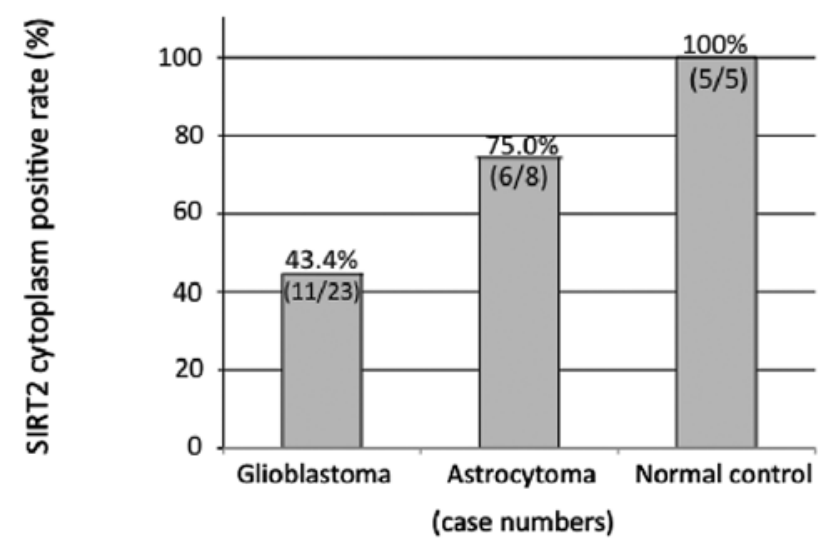

Figure 4. Nuclear and cytoplasmic SIRT2 expression in glioblastoma, astrocytoma, and normal brain samples. (A) Nuclear SIRT2 expression is shown as SIRT2 labeling index (LI). SIRT2-LI in GB samples was significantly higher than that in normal control samples ( ${ }^{*} \mathrm{P}<0.01$, Mann-Whitney's U-test) and was significantly higher than that in diffuse astrocytoma samples $\left({ }^{* *} \mathrm{P}<0.05\right)$. (B) SIRT2 cytoplasm-positive rate was lower in the highest grade gliomas. N.S., not significant.

$65.8 \pm 18.6$ for GB (grade IV) specimens, $41.2 \pm 22.8$ for DA (grade II) specimens, and $28.6 \pm 12.3$ for normal control specimens (mean \pm SD, Fig. 4A and Table I). The mean SIRT2-LI of the GB specimens was significantly higher than that of normal control specimens ( $\mathrm{P}=0.003$, Mann-Whitney's U-test) and significantly higher than that of DA specimens $(\mathrm{P}=0.021)$ (Fig. 4A and Table I). However, there was no significant difference in mean SIRT2-LI between DA and normal control specimens $(\mathrm{P}=0.31)$. Conversely, SIRT2 cytoplasm positive rate was $43.4 \%$ for GB specimens (11/23), $75.0 \%$ DA specimens (6/8), 100\% normal control specimens (5/5) (Fig. 4B and Table I). In this analysis, SIRT2 nuclear localization was observed more frequent in the more malignant specimens, and, conversely, cytoplasmic localization was less frequent in the more malignant samples (Fig. 5).

Prognostic significance of SIRT2-LI for glioblastoma. In general, it seemed that SIRT2-LI value was negatively related to survival time in patients with glioma (Table I). To evaluate the prognostic significance of the SIRT2-LI and this apparent relationship, the samples from patients with GB were divided in two groups, low SIRT-LI $(<60 \%, \mathrm{n}=7)$ and high SIRT2-LI $(\geq 60 \%, n=16$ ), and survival curve of the patients represented in each group was calculated using the Kaplan-Meier method and log-rank test. The patients represented in the low SIRT2-LI group had a significantly longer survival time than the patients represented in the high SIRT2-LI group (Fig. 6, P<0.05, KaplanMeier method and log-rank test). These findings indicated that SIRT2-LI might be a useful marker for the prognosis of GB patients.

\section{Discussion}

Glioma is the most common brain tumor in humans, and it represents $\sim 25 \%$ of primary brain tumors. According to WHO International Histological Classification of Tumors, glioma is divided into four grades based on histology (19). High grade glioma, glioblastoma (GB, grade IV), is the most malignant and has a median survival time of $\sim 1$ year, even after surgical resection, radiation therapy, and chemotherapy. By contrast, patients with low-grade DA (grade II) have a better prognosis and a median survival time of 10-15 years (21). To develop new and useful prognostic markers for GB, it is necessary to understand more precisely the process of gliomagenesis. The proportion of tumor cells with abnormal p53 protein expression increases in gliomas as they undergo malignant progression $(7,22)$. As in the case of $\mathrm{p} 53$, aberrant SIRT2 protein expression may contribute to malignant progression in glioma.

Reportedly, SIRT2 protein mainly localizes to the nucleus during the mitotic phase of the cell cycle in normal cells, and the protein mainly localized to the cytoplasm during all other phases of the cell cycle (10). In neoplastic tissues, the percentage of cells showing mitotic phase increases according to malignancy progresses. Thus, the high SIRT2-LI and the low SIRT2 cytoplasm positive rate in GB samples might have reflected a larger percentage of cells in mitosis in gliomas.

SIRT2 protein mainly localizes to the centrosome in nucleus of the HeLa cells (10). Moreover, overexpression of SIRT2 in the nucleus of HeLa cells causes multinucleation (10). Based on these observations, we suggest that nuclear accumulation of SIRT2 in glioma might cause multinucleation, a morphological marker of malignancy in gliomas. In cytoplasm of SAOS2 cells, SIRT2 protein binds to histone deacetylase 6 (HDAC6) (23), and activation of cytoplasmic HDAC6 is reportedly related to oncogenic tumorigenesis (24). Moreover, a SIRT2 and HDAC6 (SIRT2-HDAC6) complex binds to the spindle apparatus at mitosis in SAOS2 cells (23). SIRT2, together with HDAC6, plays a role in regulating microtubule dynamic instability and the deacetylation of tubulin to control progression of mitotic phase (23). Therefore, the high SIRT2-LI and low SIRT2 cytoplasm positive rate that we observed in some glioma samples might have reflected the phenomena of tumorigenesis itself, and SIRT2 protein might translocate from cytoplasm to the nucleus as gliomas become more malignant.

The immunohistochemical determination of proliferative activity using the monoclonal antibody MIB-1, which recognizes Ki-67 a nuclear antigen, has been widely demonstrated to be clinically useful in distinguishing malignancies from 
A. Normal astrocyte
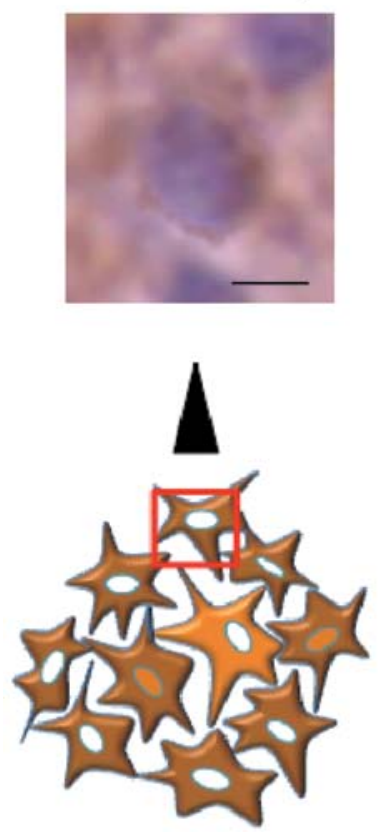

B. Astrocytoma
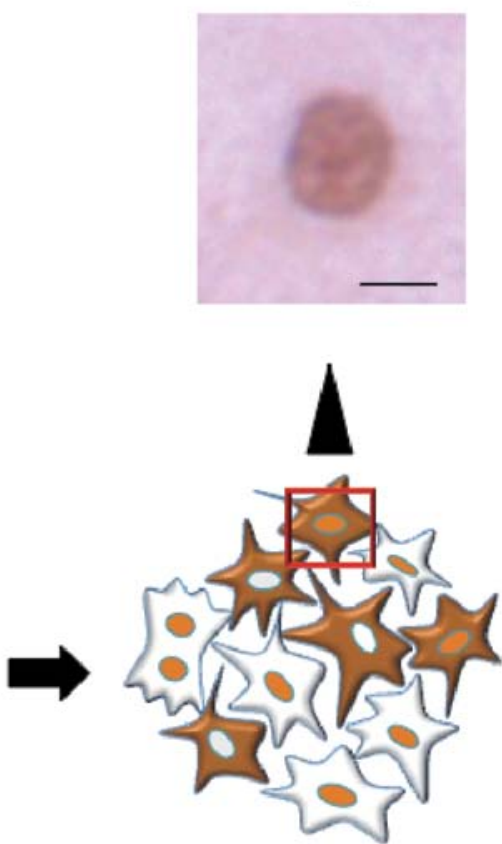

C. Glioblastoma
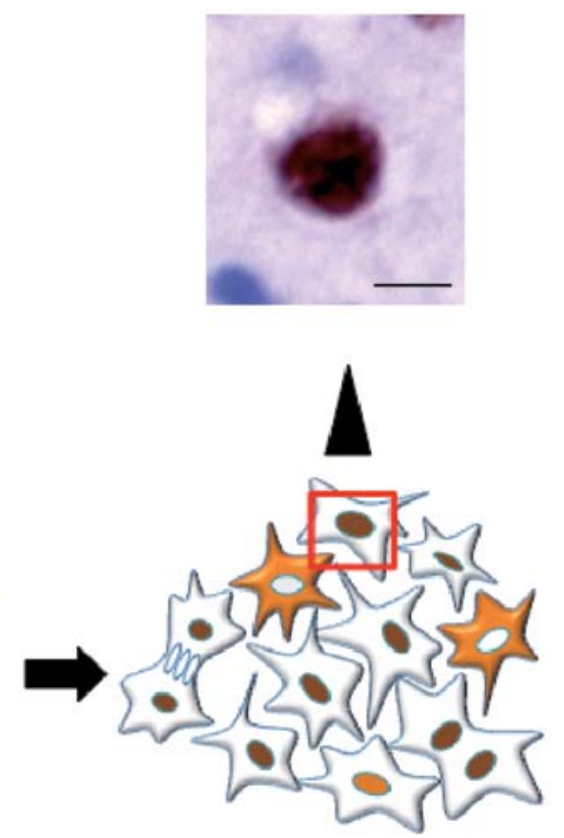

Figure 5. Micrographs and schematic illustrations of SIRT2 subcellular localization and intensity in normal astrocyte, diffuse astrocytoma (DA), and glioblastoma (GB). (A) Normal astrocyte: SIRT2 was mainly localized in cytoplasm of normal astrocytes. (B) DA: SIRT2 was localized in nuclei and cytoplasm of DA cells. (C) GB: SIRT2 was mainly localized in nuclei of GB cells. Moreover, the staining intensity of nuclear SIRT2 in GB cells was greater than that in DA cells. SIRT2 localization to the nucleus increased as malignancies progress and, conversely, cytoplasmic localization decreases as malignancies progress. Bar, $10 \mu \mathrm{m}$.

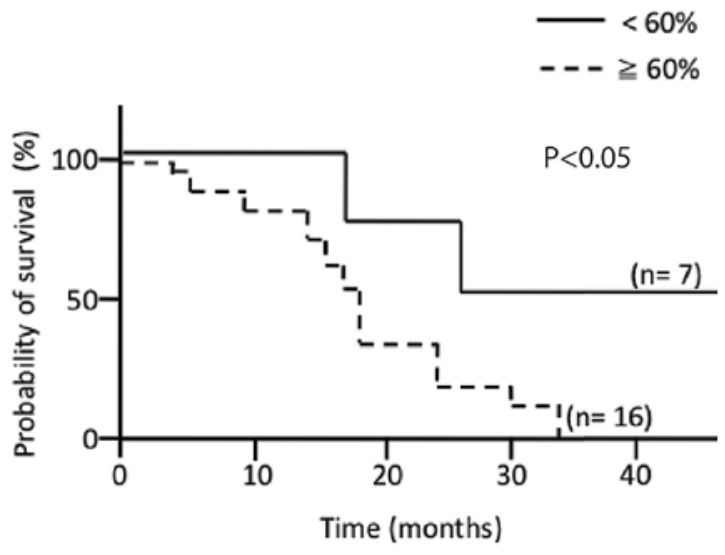

Figure 6. Survival curve analyzed by Kaplan-Meier method and log-rank test. Samples from patients with GB were separated into two groups, low SIRT2-LI $(<60 \%, n=7)$ and high SIRT2 $(\geq 60 \%, n=16)$, and then survival curves of the two groups were analyzed using the Kaplan-Meier method and log-rank test. The low SIRT2-LI group had a significantly longer survival time than the SIRT2-LI high group $(\mathrm{P}<0.05)$.

benign tumor cells $(25,26)$. However, MIB-1-LI did not reliability correlate with patient survival in cases of GB (27). To date, there are few established diagnostic markers for GB or useful prognostic markers for patients with GB $(28,29)$, although expression of WT1 (Wilms' tumor gene) and nestin, and IDH-1/2 gene mutation are reported as diagnostic or prognostic markers $(30,31)$. Our study demonstrated that SIRT2-LI was a marker of malignancy for GB and that SIRT2-LI was significantly correlated with the survival time of patients with $\mathrm{GB}$, indicating that SIRT2-LI could predict the prognosis of GB patients.

\section{Acknowledgements}

We thank Ms. Atsuko Iwata and Ms. Tomomi Araoka (Divisions of Neuropathology, Department of Brain and Neurosciences, Tottori University Faculty of Medicine) for their excellent technical assistance.

\section{References}

1. Huse JT and Holland EC: Targeting brain cancer: advances in the molecular pathology of malignant glioma and medulloblastoma. Nat Rev Cancer 10: 319-331, 2010.

2. Frederick L, Wang XY, Eley G and James CD: Diversity and frequency of epidermal growth factor receptor mutations in human glioblastomas. Cancer Res 60: 1383-1387, 2000.

3. Shete S, Hosking FJ, Robertson LB, et al: Genome-wide association study identifies five susceptibility loci for glioma. Nat Genet 41: 899-904, 2009.

4. Wrensch M, Jenkins RB, Chang JS, et al: Variants in the CDKN2B and RTEL1 regions are associated with high-grade glioma susceptibility. Nat Genet 41: 905-908, 2009.

5. Di Rocco F, Carroll RS, Zhang J and Black PM: Platelet-derived growth factor and its receptor expression in human oligodendrogliomas. Neurosurgery 42: 341-346, 1998.

6. Xiao A, Wu H, Pandolfi PP, Louis DN and Van Dyke T: Astrocyte inactivation of the $\mathrm{pRb}$ pathway predisposes mice to malignant astrocytoma development that is accelerated by PTEN mutation. Cancer Cell 1: 157-168, 2002.

7. Ohgaki $\mathrm{H}$ and Kleihues P: Genetic pathways to primary and secondary glioblastoma. Am J Pathol 170: 1445-1453, 2007.

8. Hiratsuka M, Inoue T, Toda T, et al: Proteomics-based identification of differentially expressed genes in human gliomas: down-regulation of SIRT2 gene. Biochem Biophys Res Commun 309: 558-566, 2003.

9. Michan S and Sinclair D: Sirtuins in mammals: insights into their biological function. Biochem J 404: 1-13, 2007.

10. North BJ and Verdin E: Interphase nucleo-cytoplasmic shuttling and localization of SIRT2 during mitosis. PLoS One 2: e784, 2007. 
11. Blander G and Guarente L: The Sir2 family of protein deacetylases. Annu Rev Biochem 73: 417-435, 2004.

12. Pandithage $\mathrm{R}$, Lilischkis $\mathrm{R}$, Harting $\mathrm{K}$, et al: The regulation of SIRT2 function by cyclin-dependent kinases affects cell motility. J Cell Biol 180: 915-929, 2008.

13. Harting K and Knoll B: SIRT2-mediated protein deacetylation: An emerging key regulator in brain physiology and pathology. Eur J Cell Biol 89: 262-269, 2010.

14. Inoue T, Nakayama Y, Yamada H, et al: SIRT2 downregulation confers resistance to microtubule inhibitors by prolonging chronic mitotic arrest. Cell Cycle 8: 1279-1291, 2009.

15. Inoue T, Hiratsuka M, Osaki M and Oshimura M: The molecular biology of mammalian SIRT proteins: SIRT2 in cell cycle regulation. Cell Cycle 6: 1011-1018, 2007.

16. Inoue T, Hiratsuka M, Osaki M, et al: SIRT2, a tubulin deacetylase, acts to block the entry to chromosome condensation in response to mitotic stress. Oncogene 26: 945-957, 2007.

17. Kato M, Brijlall D, Adler SA, Kato S and Herz F: Effect of hyperosmolarity on alkaline phosphatase and stress-response protein 27 of MCF-7 breast cancer cells. Breast Cancer Res Treat 23: 241-249, 1992.

18. Louis DN, Ohgaki H, Wiestler OD, et al: The 2007 WHO classification of tumours of the central nervous system. Acta Neuropathol 114: 97-109, 2007.

19. Fukuoka J, Fujii T, Shih JH, et al: Chromatin remodeling factors and BRM/BRG1 expression as prognostic indicators in non-small cell lung cancer. Clin Cancer Res 10: 4314-4324, 2004.

20. Werner HB, Kuhlmann K, Shen S, et al: Proteolipid protein is required for transport of sirtuin 2 into CNS myelin. J Neurosci 27: 7717-7730, 2007

21. Holland EC: Gliomagenesis: genetic alterations and mouse models. Nat Rev Genet 2: 120-129, 2001.
22. Zhu Y, Guignard F, Zhao D, et al: Early inactivation of $\mathrm{p} 53$ tumor suppressor gene cooperating with NF1 loss induces malignant astrocytoma. Cancer Cell 8: 119-130, 2005.

23. Nahhas F, Dryden SC, Abrams J and Tainsky MA: Mutations in SIRT2 deacetylase which regulate enzymatic activity but not its interaction with HDAC6 and tubulin. Mol Cell Biochem 303 221-230, 2007.

24. Lee YS, Lim KH, Guo X, et al: The cytoplasmic deacetylase HDAC6 is required for efficient oncogenic tumorigenesis. Cancer Res 68: 7561-7569, 2008.

25. Schiffer D, Cavalla P, Chio A, Richiardi P and Giordana MT: Proliferative activity and prognosis of low-grade astrocytomas. J Neurooncol 34: 31-35, 1997

26. Di X, Nishizaki T, Harada K, Kajiwara K, Nakayama H and Ito H: Proliferative potentials of glioma cells and vascular components determined with monoclonal antibody MIB-1. J Exp Clin Cancer Res 16: 389-394, 1997.

27. Uematsu M, Ohsawa I, Aokage T, et al: Prognostic significance of the immunohistochemical index of survivin in glioma: a comparative study with the MIB-1 index. J Neurooncol 72 231-238, 2005.

28. Johannessen AL and Torp SH: The clinical value of Ki-67/ MIB-1 labeling index in human astrocytomas. Pathol Oncol Res 12: 143-147, 2006

29. Moskowitz SI, Jin T and Prayson RA: Role of MIB1 in predicting survival in patients with glioblastomas. J Neurooncol 76: 193-200, 2006.

30. Rushing EJ, Sandberg GD and Horkayne-Szakaly I: High-grade astrocytomas show increased Nestin and Wilms' tumor gene (WT1) protein expression. Int J Surg Pathol 18: 255-259, 2010.

31. Ducray F, Idbaih A, Wang XW, Cheneau C, Labussiere M and Sanson M: Predictive and prognostic factors for gliomas. Expert Rev Anticancer Ther 11: 781-789, 2011. 